\title{
O REGIME DE INFORMAÇÃO DA COMUNICAÇÃO CIENTÍFICA: UMA ABORDAGEM
}

\section{THE SCIENTIFIC COMMUNICATION INFORMATION REGIME: AN APPROACH}

\author{
Gustavo Henrique de Araujo Freirea
}

\begin{abstract}
RESUMO
Objetivos: Tem como objetivo geral identificar a literatura que aborda ações de informação desenvolvidas no ciberespaço através de redes sociais de periódicos para comunicação e divulgação do conhecimento científico para a sociedade. Os objetivos específicos são: mapear a produção da área de Ciência da Informação que aborda as redes sociais na comunicação e divulgação científica; descrever a rede de periódicos e os descritores que formam o escopo epistemológico da comunicação e divulgação científica, e redes sociais; apresentar o regime de informação que envolve a comunicação e divulgação científica. Metodologia: A pesquisa é exploratória, descritiva, e de natureza qualitativa, utilizando técnicas de pesquisa documental, organizando os resultados a partir de categorias para alcançar os objetivos traçados. Resultados esperados: Espera-se propiciar uma contribuição para o enriquecimento dos estudos da temática na Ciência da Informação, bem como a compreensão de como o processo de comunicação e divulgação científica está utilizando esse novo canal de comunicação, na dimensão técnica, social e econômica. Esse processo poderá revelar possibilidades, limitações e aplicações futuras das redes sociais digitais para o periódico científico, não só no contexto da Ciência da Informação como, também, em outras áreas do conhecimento científico.
\end{abstract}

Descritores: Comunicação científica. Divulgação científica. Redes sociais digitais. Revisão de literatura.

\section{INTRODUÇÃO}

O artigo aborda temática relevante nos estudos dos processos de comunicação da informação, no escopo do campo científico da Ciência da Informação, qual seja a comunicação científica e as redes sociais. Nesse sentido, nossa proposta apresenta uma abordagem que leva a uma reflexão

\footnotetext{
a Doutor em Ciência da Informação pela Universidade Federal do Rio de Janeiro (UFRJ). Docente do Curso de Biblioteconomia e Gestão de Unidades de Informação Universidade Federal da Paraíba (PPGCI-UFPB). Docente do Programa de Pós-Graduação em Ciência da Informação da Universidade Federal do Rio de Janeiro (IBICT/UFRJ). E-mail: gustavofreire@facc.ufrj.br
} 
acerca das mudanças recentes ocorridas no já consolidado sistema de comunicação científica, observando sua dimensão social implementada através da divulgação científica, com ações de informação voltadas para a democratização da ciência, assim como os novos espaços informacionais onde as informações são compartilhadas/publicizadas. Os novos espaços infocomunicacionais são representados pelas redes sociais digitais, que estão se tornando espaços relevantes para a produção e comunicação da informação.

Nossa reflexão irá ocorrer a partir dos resultados alcançado a partir dos seguintes objetivos específicos: mapear a produção científica da área de Ciência da Informação que aborda as redes sociais na comunicação científica; descrever a rede de periódicos e os descritores que compõem o escopo epistemológico da comunicação científica e redes sociais; apresentar o regime de informação que envolve a comunicação científica.

Neste contexto, a sociedade contemporânea tem, nas tecnologias digitais de informação e comunicação, a par do uso intensivo de informação e conhecimento, a tríade que direciona um novo ordenamento social, econômico e informacional que faz com que o regime de informação seja hegemônico. Assim, as principais atividades sociais e econômicas estão organizadas a partir da geração, organização e comunicação de informações em suporte digitais e em formato de rede.

Segundo Castells (2015), uma sociedade em rede é uma sociedade cuja estrutura social é construída em torno de redes ativadas por tecnologias de comunicação e de informação processadas digitalmente e baseadas na microeletrônica. Nesse contexto, a sociedade contemporânea tem na informação a matéria-prima para o desenvolvimento social e econômico, e nas redes digitais de comunicação e informação os meios que tornam possível a sua disseminação com rapidez e penetrabilidade em todas as dimensões da sociedade.

Destarte, se na sociedade contemporânea é importante para o indivíduo comum ter acesso e compartilhar informações, para pesquisadores, professores e estudantes trata-se de questão fundamental, pois é através do acesso e das trocas informacionais que novos conhecimentos são criados. Contudo, estudar informação nas redes sociais é um grande desafio, na medida em que são 
abordados conceitos dinâmicos que evoluem e se moldam em variados contextos. Segundo Marteleto (2010, p. 33):

Face às mutações, permanências e remetimentos que as noções de rede e de informação colocam na arena de discussões da sociedade da informação, é relevante observar que tanto uma quanto a outra são noções transversais. Portanto, são complexas, uma vez que integram os dispositivos técnicos, as representações humanas e sociais, as escolhas políticas e a hegemonia econômica e científica dos espaços e tempos mundializados.

Nesse contexto, a informação se destaca como um bem intangível de grande valor para a sociedade, especialmente no campo científico. Ao longo do tempo ocorreu um crescimento exponencial de informações científicas e acadêmicas, e também da diversidade nos espaços voltados para armazenagem, organização e comunicação da informação, principalmente no contexto das tecnologias digitais de comunicação e informação. No contexto da comunicação e divulgação científica surgem novos canais, as redes sociais digitais, que podem colaborar com os periódicos científicas em vários aspectos, dos quais destacamos o aumento de acesso ao periódico o que leva à maior visibilidade, o compartilhamento de informações em diferentes plataformas, que implica em diferentes linguagens e diferentes usuários, e a possibilidade de aproximação dos processos de comunicação e divulgação científica, facilitada pelo uso das tecnologias digitais de informação e comunicação.

A nossa abordagem se fundamenta, parafraseando Wersig e Neveling (1975), na nova relevância de um fenômeno antigo, visto que a configuração em rede sempre ocorreu na história humana e foi fundamental para o seu desenvolvimento e evolução. Contudo, na contemporaneidade, com as tecnologias digitais, as redes sociais ganham nova relevância, especialmente 0 papel das redes sociais digitais na comunicação científica no contexto da sociedade contemporânea.

Nesse sentido, a penetrabilidade e o uso intensivo das tecnologias digitais de informação e comunicação tornaram-se fundamentais para a constituição das redes de comunicação científica que caracterizam a sociedade contemporânea, já que essas redes são utilizadas por milhões de usuários e ganham cada vez 
mais protagonismo no ambiente científico.

\section{SOBRE COMUNICAÇÃO CIENTÍFICA NO REGIME DE INFORMAÇÃO DA SOCIEDADE EM REDE}

Na Ciência da Informação, o conceito de Regime de Informação vem sendo apresentado e discutido por diversos autores que o utilizam como fio condutor de suas pesquisas. Nesse contexto, Frohmann (1995) utiliza o conceito em uma perspectiva tecnológica, focando nos canais de comunicação; Braman (2004), por sua vez, apresenta o regime de informação de alcance global, relacionado às políticas de informação, ao poder do Estado informacional e à penetrabilidade das tecnologias digitais de informação e comunicação.

Para fins desta pesquisa, adotamos o conceito de regime de informação de González de Gómez, pois a autora, além de pioneira no Brasil nos estudos da temática, acrescenta às abordagens supracitadas a relação com conceitos de gestão e políticas de informação, além de incluir, no ambiente composto pelo regime de informação, todos os elementos que representam a sociedade contemporânea.

Nesse sentido, apresenta a oportunidade para que se estude a sociedade em todas as suas dimensões na perspectiva do regime de informação, visto que para González de Gómez a sociedade contemporânea é aquela em que o regime de informação é hegemônico. Segundo a autora,

A sociedade da informação poderia ser entendida como aquela
em que o regime de informação caracteriza e condiciona todos
os outros regimes sociais, econômicos, culturais, das
comunidades e do estado. Nesse sentido, a centralidade da
comunicação e da informação produziria a maior dispersão das
questões políticas da informação, perpassada e interceptada por
todas as outras políticas: as públicas e as informais, as tácitas e
as explícitas, as diretas ou as indiretas. (GONZÁLEZ DE
GÓMEZ, 1999, p. 2).

Em nossa abordagem, entendemos o regime de informação como um conceito metodológico-relacional-pragmático que auxilia na visão de um determinado contexto, facilitando a visão geral através da identificação de seus elementos. Para fins desta pesquisa, iremos discorrer sobre três 
elementos do regime de informação, que são os que mais nos interessam, quais sejam os atores sociais, os artefatos de informação e os dispositivos de informação.

Os atores sociais são responsáveis pela geração e recepção de informações no regime de informação, e os dividimos em duas categorias, humanos e não humanos, estes representados pelas instituições às quais os atores sociais humanos estão vinculados. No processo de comunicação científica encontramos como atores sociais humanos, os produtores de informação em potencial, como pesquisadores, docentes, especialmente os que estão vinculados a Programas de Pós-Graduação; os discentes com seus trabalhos de final de curso de graduação, dissertações e teses. Também fazem parte deste grupo os atores sociais que desenvolvem ações de informação relacionadas às atividades de gestão da produção das informações. Nesta categoria estão os Coordenadores de Programas de PósGraduação, Líderes de Grupos de Pesquisa, Editores de periódicos científicos.

Os atores não humanos são representados por universidades, grupos de pesquisa, programas de pós-graduação, associações e periódicos científicos, que constituem uma comunidade específica de produtores de informação. Podem ser considerados como atores sociais de informação na medida em que têm a sua produção científica contabilizada pelas agências de fomento e de controle da produção científica. O resultado das análises de produção de atores não humanos apresenta resultados similares às avaliações dos atores humanos, tais como prestígio e maior acesso aos recursos de agências de fomentos à pesquisa, entres outros indicadores importantes para o ambiente de produção e comunicação da ciência 
Figura 1 - Atores humanos e não humanos

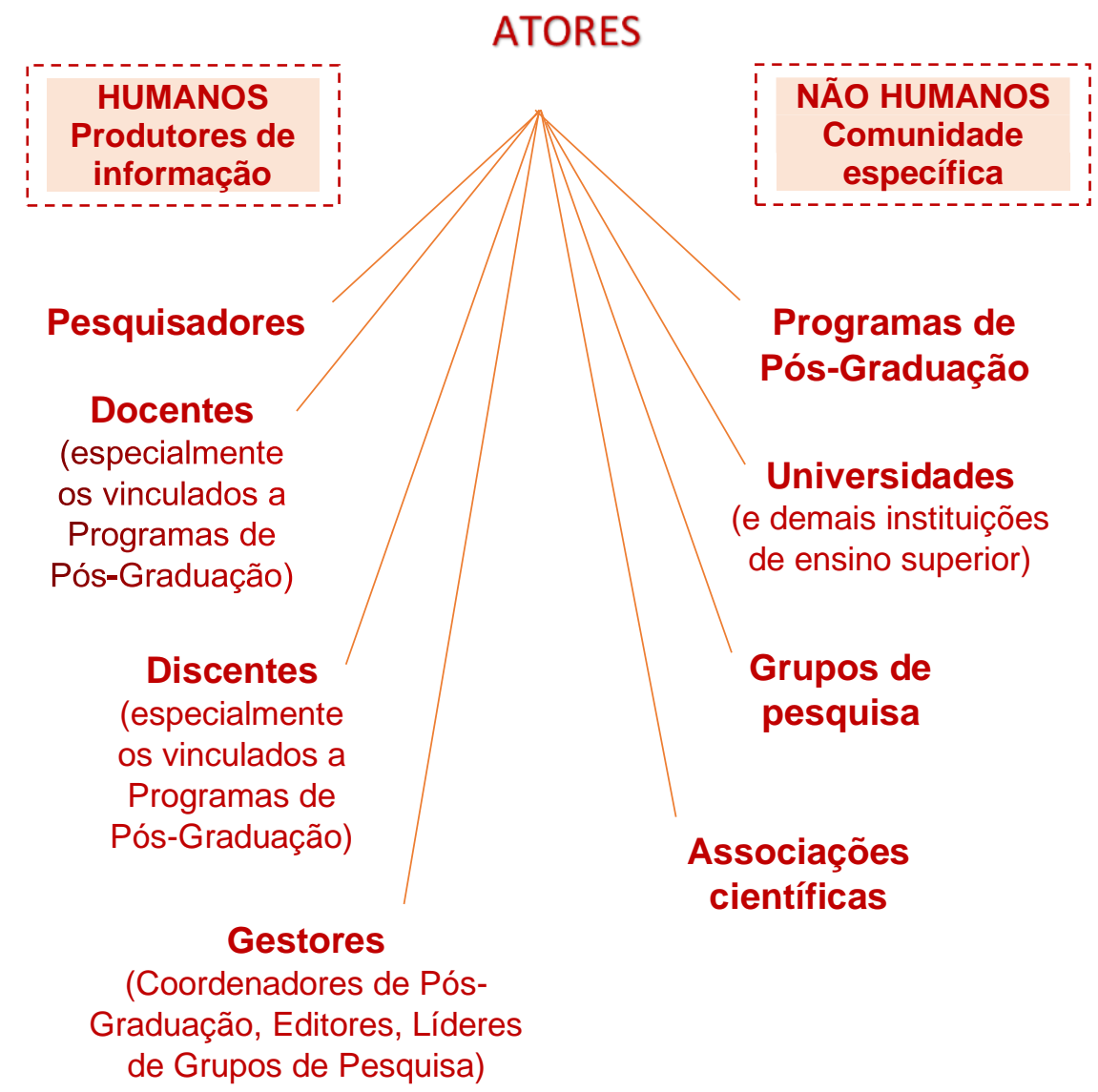

Fonte: Elaboração própria, 2021

Os artefatos de informação são responsáveis pelos espaços em que as informações estão inseridas, organizadas e disponibilizadas. Podem ser observados em duas dimensões, uma tecnológica e outra semântica. A dimensão tecnológica está relacionada aos suportes que permitem 0 armazenamento das informações, que denominamos Artefato Informacional Sintático (AISint), onde predominam a possibilidade e necessidade de relações convergentes que permitem a interoperabilidade entre as variadas plataformas tecnológicas para geração, organização e comunicação de informações.

É importante ressaltar que estes artefatos de informação que estamos denominando A/Sint, no âmbito do regime de informação da comunicação científica, estão relacionados aos canais de comunicação por onde circulam as informações. Aos canais tradicionais utilizados pelos atores sociais para comunicação de suas pesquisas, tais como como periódico científico, livros e 
eventos científicos, foram incorporados novos AISint, especialmente os que apresentam inovações tecnológicas relacionadas aos novos espaços infocomunicacionais proporcionados pela emergência das tecnologias digitais de informação e comunicação, que possibilitaram a emergência do que Castells denomina sociedade em rede. Neste contexto surgiram as redes sociais digitais no campo da comunicação científica, que são plataformas voltadas para comunicação em rede, como Twitter, Instagam e Facebook, e que estão ocupando um espaço especial na comunicação de informação científica.

A outra dimensão dos artefatos de informação está relacionada ao conteúdo das informações, à qual denominamos Artefato Informacional Semântico (AIS). Esta dimensão está voltada para os variados tipos de informações que se podem encontrar nos AISint, tais como artigos (e suas variadas temáticas e tipologias ), preprints, livros, palestras, ou seja, toda uma extensa gama de tipos de informação que fazem parte do processo de compartilhamento dos resultados de pesquisas, e que tem no processo de comunicação científica o ambiente informacional por excelência para a sua comunicação entre pares e, posteriormente, para toda a sociedade através de ações para a democratização da ciência, como a divulgação científica.

Figura 2 - Artefatos de informação

\section{ARTEFATOS DE INFORMAÇÃO}

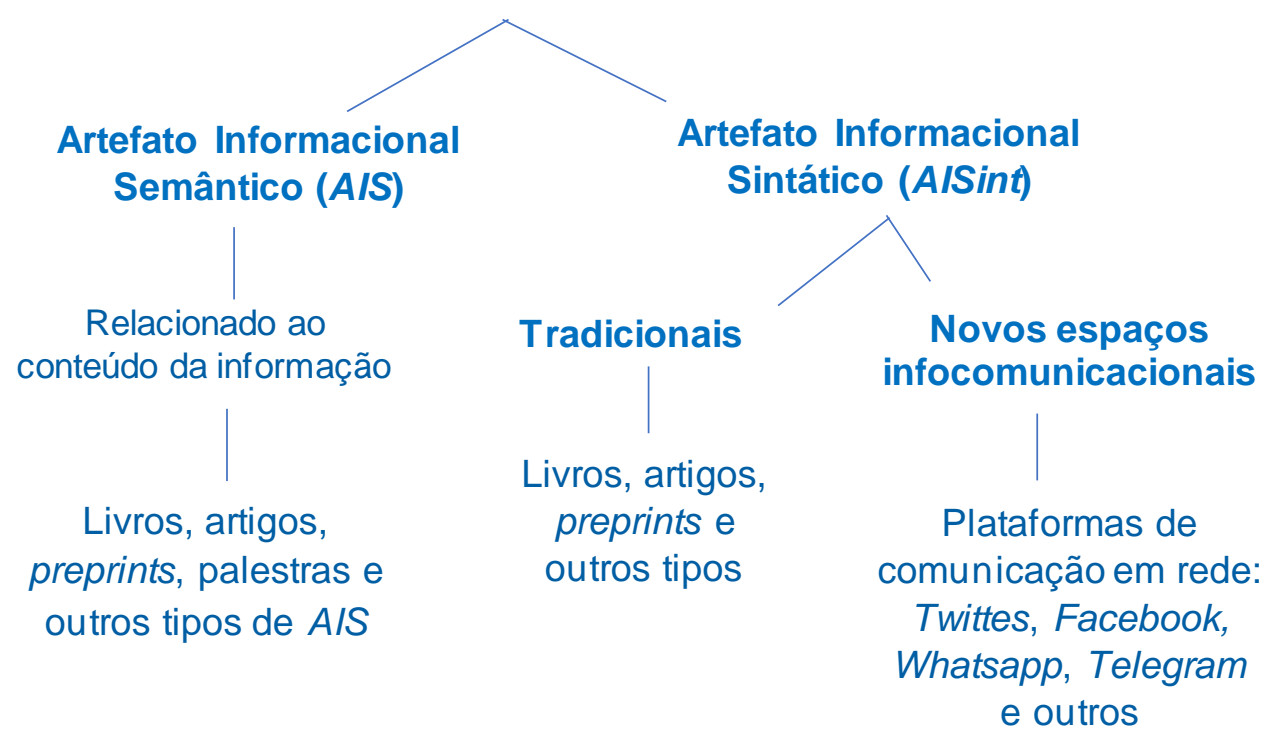

Fonte: Elaboração própria, 2021 
Por sua vez, dispositivos de informação são os elementos responsáveis pelas normas e regras que regem um regime de informação. No caso específico da nossa abordagem, o regime da comunicação científica. $O$ processo de comunicação científica vem sendo construído através de séculos e se baseia no desenvolvimento de regras que se articulam a partir de quatro pilares do arcabouço científico e tecnológico: (i) mérito, (ii) qualidade científica, (iii) inovação e (iv) avaliação. Nesse sentido, consideramos como dispositivos de informação, no âmbito da comunicação científica, as normas para participação em eventos científicos, as regras para publicação em periódicos, visto que as revistas científicas devem apresentar suas políticas de informação, bem como seus templates visando a padronização do formato dos artigos. Observamos, ainda, os dispositivos de informação que orientam e normalizam os processos para o desenvolvimento de projetos de pesquisa, assim como para solicitação de recursos para pesquisas, visando a obtenção de bolsas, equipamentos e financiamento de custeio. Essas regras não são uniformes, mas seguem os quatro pilares supracitados.

Figura 3 - Dispositivos de informação

\section{DISPOSITIVOS DE INFORMAÇÃO}

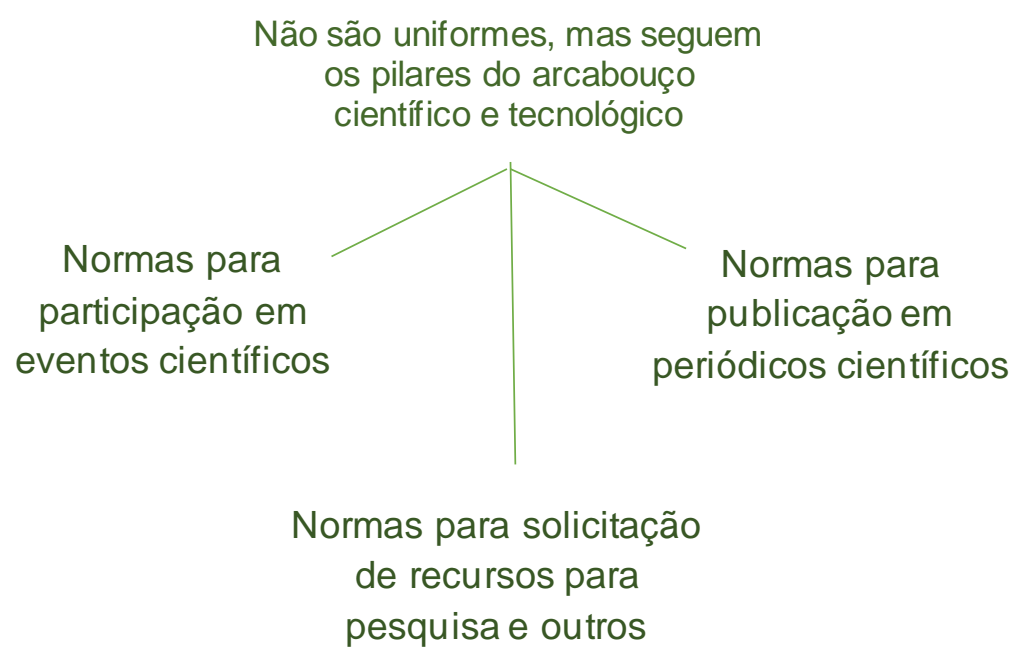

Fonte: Elaboração própria, 2021

Ao observar cada elemento do regime de informação, suas relações e categorias internas, podemos identificar o universo onde atuam produtores e usuários de informação, as normas e regras que norteiam o espaço 
informacional, a organização e criação de informações em artefatos informacionais, bem como as ações de informação que ocorrem no ambiente informacional, especialmente as ações formativas, que se relacionam com os aspectos socioculturais, pois a comunidade que faz parte do contexto da comunicação científica apresenta traços culturais e sociais específicos, como também necessidades informacionais próprias.

Voltando à abordagem de González de Gómez (1999, p. 27), corroboramos sua definição de regime de informação como

[...] um conjunto de redes formais e informais nas quais as informações são geradas, organizadas e transferidas de diferentes produtores, através de muitos e diversos meios, canais e organizações a diferentes destinatários ou receptores de informação, sejam esses usuários específicos ou públicos amplos. Em síntese, um regime de informação se caracteriza por sua complexidade e sua não transparência imediata, por nele ocorrerem conflitos, vontades plurais e efeitos não desejados.

É nessa perspectiva iremos apresentar o regime de informação do ambiente informacional da comunicação científica, identificando os elementos do regime de informação nesse sistema, que já tem mais de 300 anos de existência e tem acompanhado as mudanças ocorridas na sociedade. Essas mudanças têm atuado em todas as fases e dimensões do processo de comunicação científica, quais sejam, informacional, gerencial e tecnológica.

Nesse sentido, apresentar o regime de informação do processo de comunicação científica é relevante pela oportunidade de compreender, através de uma perspectiva holística, todo o ambiente informacional que compõe o que podemos denominar de um dos grandes saltos qualitativos na história da humanidade, conforme Santillana (1970): a criação da atividade científica, e, como consequência, o processo de comunicação científica.

Compartilhar o resultado das pesquisas com a comunidade científica sempre foi e continuará sendo, a nosso ver, o principal objetivo para todos que atuam no campo científico. O compartilhamento dos resultados do trabalho de pesquisa, através de sua publicização, permite que o conhecimento científico seja divulgado e que novos conhecimentos sejam gerados. Nesse contexto, o periódico científico é um AISint que, desde a sua origem, tem acompanhado as inovações tecnológicas, gerenciais e comunicacionais que permeiam o processo 
de comunicação científica. Apesar do surgimento de novos espaços infocomunicacionais trazidos pelo surgimento das plataformas de comunicação em rede, o periódico continua sendo o principal canal para a comunicação do resultado das pesquisas científicas.

A criação de novos espaços de comunicação de caráter científico, convivendo com os tradicionais periódicos, é resultado da dinâmica da comunicação social contemporânea, em que o regime de informação dominante exige novas formas de expressão e compartilhamento da informação, as quais crescem exponencialmente e demandam novos canais para sua disseminação. Por sua vez, esses novos meios exigem estratégias e mecanismos próprios para disponibilização e busca de informação, apresentando, sem embargo, alguns problemas que acreditamos serão solucionados ou redimensionados com 0 tempo.

Neste contexto, os usuários que fazem parte da comunidade científica têm à disposição novos artefatos informacionais sintáticos para disponibilizar o resultado de suas pesquisas. É um cenário novo, em rápida transformação, que merece ser estudados por sua relevância, inovação e novas possiblidades para comunicação científica. A sinergia, convergência de linguagens e relações entre variadas plataformas devem ser observadas para que se possa atingir todo 0 seu potencial e colaborar com o principal objetivo do processo de comunicação científica, qual seja, a geração de novos conhecimentos.

\section{COMUNICAÇÃO CIENTÍFICA E REDES SOCIAIS}

A necessidade de compartilhar os resultados das pesquisas é um ponto central do trabalho científico. O desenvolvimento da ciência ocorre porque os resultados das pesquisas são compartilhados com os pares. Esse processo envolve atores (pesquisadores, professores, estudantes), os fluxos de informação (gestão, organização da informação) e as tecnologias intelectuais voltadas para a comunicação da informação (livros, periódicos, eventos científicos). Esse processo é o que se denomina comunicação científica.

Esse modelo vem se modificando ao longo dos séculos e tem como marco a criação das primeiras associações científicas, e posteriormente a criação de 
novos canais para comunicação da informação, em especial a criação do periódico científico. As tecnologias de informação sempre foram importantes nesse processo. $\mathrm{Na}$ sociedade contemporânea as tecnologias digitais de informação e comunicação, com sua permeabilidade e alcance na sociedade, são responsáveis por grandes inovações. Como maior exemplo da inovação no processo de comunicação científica temos o periódico, que incorporou inovações tecnológicas ao passar do mundo analógico para o digital. Segundo Larivière (2015, p. 1. Tradução nossa),

Desde o século XVII, a importância dos periódicos para divulgar os resultados da pesquisa científica aumentou consideravelmente. Depois de coexistir com correspondências, monografias e tratados - que muitas vezes levaram vários anos para serem publicados - eles se tornaram, no início do século XIX, a maneira mais rápida e conveniente de divulgar novos resultados de pesquisa e seu número cresceu exponencialmente. Durante o século $X X$, eles consolidaram sua posição como a principal mídia para difundir a pesquisa , especialmente nas ciências naturais e médicas [9]. Os periódicos acadêmicos também contribuíram para a profissionalização das atividades científicas, delimitando a fronteira entre a ciência popular e a frente de pesquisa e, consequentemente, aumentando o nível de especialização da pesquisa e a formação de disciplinas.

Essas mudanças ocorrem em todas as dimensões que envolvem a comunicação científica. Os pesquisadores também modificaram as suas formas de acessar e disponibilizar os resultados de suas pesquisas e, também, de se relacionarem com seus pares.

O relacionamento entre os pesquisadores sempre foi fundamental para 0 desenvolvimento das pesquisas. Assim, as redes sociais que envolvem o modelo de comunicação científica sempre estiveram presentes no trabalho científico. Primeiramente, redes sociais presenciais em eventos científicos ou nas reuniões patrocinadas pelas associações científicas. Depois, à distância, com a troca de cartas e a criação dos Colégios Invisíveis. Atualmente, as redes sociais digitais de comunicação surgem como uma inovação sem precedentes no contexto da comunicação científica. Pela quantidade de usuários que reúnem, pela 
capacidade de colaborar com os periódicos científicas na gestão e comunicação de grande volume de informação em circulação e, especialmente, pela oportunidade de utilização de diversas plataformas, tais como blogs, Facebook, Twitter, Instagram, que podem ajudar na autonomia, acesso e compartilhamento dos resultados de pesquisas.

Trata-se também de respostas à questões mais complexas que estão relacionadas a circulação da ciência, reconfiguração do trabalho acadêmico e mudanças sobre o paradigma da comunicação para além de determinismos tecnológicos. Em um modelo de comunicação pós-massiva, baseado em um processo conversacional aberto de muitos para muitos, o controle do fluxo da informação não se limita apenas ao sujeito que detinha os poderes tradicionais decisórios sobre o que ia se tornar publicável, como no modelo tradicional. Neste novo paradigma da comunicação, diferentes formas de produção de conteúdo autônomas emergem e se reconfiguram, disputando espaço e legitimidade sobre a informação nos ambientes digitais. (OLIVEIRA, 2018, p. 3).

Nesse contexto, as redes sociais digitais podem ser vistas como aliadas dos periódicos científicos e se tornam centrais no processo de inovação na comunicação científica. A configuração em rede está estreitamente ligada à evolução humana e é própria do ser humano que se agrupa com seus semelhantes e estabeleceu relações sociais fundamentais para 0 desenvolvimento da humanidade. O sucesso da história humana está relacionado diretamente à capacidade de trabalho, ao desenvolvimento das relações em um determinado grupo, fazendo trabalho cooperativo e gerando novos conhecimentos. Nesse sentido, a configuração humana, através de redes sociais, é antiga e, até hoje, fundamental para o compartilhamento da informação e do conhecimento.

\section{METODOLOGIA E RESULTADOS}

Trabalhamos com o modelo de rede conceitual de Wersig (1993), que propõe para a Ciência da Informação a construção de estruturas teóricas que considerem mais as estratégias de ação do que a formulação de leis gerais. Nesse modelo, o quadro de referência teórico se constitui a partir de atratores conceituais, conceitos-chave que atraem materiais teóricos ou empíricos de 
outros campos científicos, "reestruturando-os dentro da estrutura científica da informação" (WERSIG, 1993, p. 231). Dessa forma, é possível tecer, no tear da Ciência da Informação, uma rede de conceitos a partir da qual abordagens teóricas e metodológicas se encontram, "fazendo a rede ainda mais inclusiva e mais apertada, de modo a aumentar seu caráter científico" (WERSIG, 1993, p. 232). Em nossa pesquisa o atrator conceitual da rede é constituído pelo conceito comunicação científica, por ser o termo fundador para definir os espaços onde a informação é compartilhada com outros cientistas.

A pesquisa documental foi desenvolvida na base de dados interdisciplinar Web of Science (WoS) da Clarivate Analytics, escolhida por sua maior abrangência, englobando, inclusive, o conteúdo da Base de Dados em Ciência da Informação (Brapci), que não possui ferramentas de análises de dados tão completas.

Utilizamos em um primeiro momento 0 argumento de busca <Comunicação Científica>, "Scientific Communication", aplicando o filtro para a categoria Information Science Library Science e definindo o recorte temporal de 2011-2021, de forma a obter um panorama geral acerca da produção em comunicação científica nesse período, e, posteriormente, a partir destes resultados, filtrarmos os dados de modo a encontrar documentos que abordam as redes sociais.

Recuperamos através do argumento, categoria e recorte temporal supracitados, o quantitativo de 296 resultados. A partir destes resultados, utilizamos a ferramenta de filtro "Procurar nos resultados..." que a base oferece para realizar uma busca utilizando, primeiramente, o termo "Social Media". Foram 38 resultados recuperados, sendo 33 artigos resultados de pesquisas, quatro artigos de conferências e um artigo de revisão. Destacando-se as produções vindas de autores dos países: Espanha (10 resultados), Brasil (8 resultados) e China (5 resultados). Nos Quadros 1 e 2 mostramos os autores e países em destaque: 


\section{Quadro 1 - Países mais produtivos}

\begin{tabular}{|c|c|}
\hline País & Quantitativo \\
\hline Espanha & 10 \\
\hline Brasil & 8 \\
\hline China & 5 \\
\hline Bélgica & 2 \\
\hline Egito & 2 \\
\hline Finlândia & 2 \\
\hline Hungria & 2 \\
\hline Turquia & 2 \\
\hline País de Gales & 2 \\
\hline
\end{tabular}

Fonte: Dados da pesquisa, 2021.

Como especial, ressaltamos a quantidade de publicações do Brasil em relação à China, por exemplo, e mesmo à Espanha, que apresenta maior número de publicações.

Quadro 2 - Autores mais produtivos

\begin{tabular}{|l|c|}
\hline \multicolumn{1}{|c|}{ Autor } & País \\
\hline Akkoyunlu B. (Cankaya University) & Turquia \\
\hline $\begin{array}{l}\text { Cetin N. M. (Adana Alparslan Turkes Science \& Technology } \\
\text { University) }\end{array}$ & Turquia \\
\hline Chen Y. (Dalian University of Technology) & China \\
\hline Ellis D. (University of Nebraska Medical Center) & EUA \\
\hline Gertrudix M. (Universidad Rey Juan Carlos) & Espanha \\
\hline Glanzel W. (Catholic University of Leuven) & Bélgica \\
\hline Maricato J. D. (Universidade de Brasília) & Brasil \\
\hline Rajas M. (Universidad Rey Juan Carlos) & Espanha \\
\hline Shehata A. (Minia University) & Egito \\
\hline Wang Z. Q. (Dalian University of Technology) & China \\
\hline
\end{tabular}

Fonte: dados da pesquisa, 2021. 
Dentre os periódicos recuperados na busca destacam-se o espanhol Profesional de La Información, com cinco resultados, e o brasileiro Em Questão, com quatro documentos recuperados. Em seguida, os demais periódicos apresentam dois resultados cada: o espanhol Anales de Documentación, o brasileiro Transinformação, o Journal of Informetrics, dos Países Baixos, e o Journal of the American Society for Information Science and Technology e Learned Publishing, dos Estados Unidos.

Quadro 3 - Periódicos mais produtivos

\begin{tabular}{|l|l|c|}
\hline \multicolumn{1}{|c|}{ Título da publicação } & \multicolumn{1}{c|}{ País } & Quantitativo \\
\hline Profesional de La Información & Espanha & 5 \\
\hline Em Questão & Brasil & 4 \\
\hline Anales de Documentación & Espanha & 2 \\
\hline Journal of Informetrics & Países Baixos & 2 \\
\hline $\begin{array}{l}\text { Journal of the American Society for } \\
\text { Information Science and Technology }\end{array}$ & EUA & 2 \\
\hline Learned Publishing & EUA & 2 \\
\hline Transinformação & Brasil & 2 \\
\hline
\end{tabular}

Fonte: Dados da pesquisa, 2021.

Analisando os registros recuperados, foi possível observarr alguns termos relacionados em destaque, tais como: "Information Literacy", e derivados, como "Networking", e o que mais se destaca, "Altmetrics", o que é de se esperar, visto que é um conceito que está intimamente ligado à temática. Dado o alto índice de aparições do termo e suas variantes, repetimos a busca utilizando os mesmos argumentos, categoria e recorte temporal, filtrando, posteriormente, com o termo altmetric ${ }^{*}$ de forma a recuperar suas variações. O resultado foi o quantitativo de 31 documentos, dentre os quais as produções brasileiras se destacam em primeiro lugar, com 15 resultados, mais que o dobro do segundo lugar, ocupado pela Espanha, com seis resultados, seguido pela China, com cinco resultados recuperados. 


\section{Quadro 4 - Países mais produtivos}

\begin{tabular}{|c|c|}
\hline País & Quantitativo \\
\hline Brasil & 15 \\
\hline Espanha & 6 \\
\hline China & 5 \\
\hline Bélgica & 3 \\
\hline Hungria & 3 \\
\hline
\end{tabular}

Fonte: Dados da pesquisa, 2021.

Também identificamos, na busca realizada, os autores mais produtivos.

Quadro 5 - Autores mais produtivos

\begin{tabular}{|l|c|c|}
\hline \multicolumn{1}{|c|}{ Autor } & País & Quantitativo \\
\hline Maricato J. D. (Universidade de Brasília) & Brasil & 4 \\
\hline Barcelos J. (Universidade de Brasília) & Brasil & 3 \\
\hline $\begin{array}{l}\text { De Araujo R. F. (Universidade Federal de } \\
\text { Alagoas) }\end{array}$ & Brasil & 3 \\
\hline Glanzel W. (Catholic University of Leuven) & Bélgica & 3 \\
\hline Alvarez-bornstein B. (CSIC - Instituto de Filosofia) & Espanha & 2 \\
\hline Chen Y. (Dalian University of Technology) & China & 2 \\
\hline Montesi M. (Complutense University of Madrid) & Espanha & 2 \\
\hline Wang Z. Q. (Dalian University of Technology) & China & 2 \\
\hline
\end{tabular}

Fonte: Dados da pesquisa, 2021.

Nessa categoria, o Brasil se destaca, com proeminência para a Universidade de Brasília. No Quadro 6, a seguir, a quantificação das revistas mais produtivas.

Quadro 6 - Revistas mais produtivas

\begin{tabular}{|l|c|c|}
\hline \multicolumn{1}{|c|}{ Título da publicação } & País & Quantitativo \\
\hline Em Questão & Brasil & 5 \\
\hline Scientometrics & Países & 3 \\
\hline
\end{tabular}




\begin{tabular}{|l|c|c|}
\hline & Baixos & \\
\hline Bibliotecas-Anales de Investigación & Cuba & 2 \\
\hline $\begin{array}{l}\text { Perspectivas em Ciência da } \\
\text { Informação }\end{array}$ & Brasil & 2 \\
\hline Profesional de La Información & Espanha & 2 \\
\hline
\end{tabular}

Fonte: Dados da pesquisa, 2021.

Repetimos a estratégia inicial, utilizando, desta vez, o argumento de busca <Divulgação Científica>, "Scientific Dissemination" que, apesar de não fazer parte do escopo inicial da pesquisa tem relação de proximidade com a comunicação científica, podendo ser considerada como uma ampliação das atividades relacionadas à comunicação científica, se diferenciando desta em relação ao público, linguagem utilizada e intencionalidade. Essa temática, também relevante para a área da Ciência da Informação, será objeto de estudo em outro momento.

A busca retornou apenas 10 resultados. Analisando os resultados, pudemos observar uma falta de padronização no uso do termo para designar o conceito, pois encontramos documentos que utilizam "Science Dissemination" e em alguns momentos é possível perceber uma certa confusão entre os conceitos de Comunicação Científica e Divulgação Científica, prejudicando nossa pesquisa.

De forma a ampliar os resultados, abarcando as variações do uso de termos para designar o conceito, utilizamos o argumento de busca "Scientific Dissemination" OR "Science Dissemination". Aplicando o filtro para a categoria Information Science Library Science, definindo o recorte temporal de 2011-2021 e buscando nos resultados o termo "social media", obtivemos o quantitativo de 11 documentos (nove artigos, um material editorial e um artigo de conferência). Por conta da escassez de resultados, foi difícil analisar a maior incidência de determinados termos, porém, foi possível perceber a relação entre termos relacionados a altimetrias e competência em informação.

Entre os autores, apenas dois se destacam com dois trabalhos cada: Gertrudix, M. e Rajas, M. Ambos os trabalhos são colaborações entre os autores. Verificamos que os dois são filiados à mesma instituição, a Universidad Rey Juan 
Carlos, da Espanha. Além disso, publicaram em uma mesma revista, Profesional de La Información, da Espanha, em números diferentes. A revista também se destaca nesse cenário, com quatro resultados recuperados. Entre os países, mais um destaque para a Espanha, com cinco resultados, seguida pelo Brasil, com quatro.

Quadro 7 - Revistas mais produtivas

\begin{tabular}{|l|c|c|}
\hline \multicolumn{1}{|c|}{ Título da Publicação } & País & Quantitativo \\
\hline Profesional de La Información & Espanha & 4 \\
\hline $\begin{array}{l}\text { Applications Of Grey Literature For Science And } \\
\text { Society }\end{array}$ & Países Baixos & 1 \\
\hline Atoz Novas Práticas em Informação e Conhecimento & Brasil & 1 \\
\hline Gl Conference Series & Países Baixos & 1 \\
\hline Informação Sociedade Estudos & Brasil & 1 \\
\hline Information Society & EUA & 1 \\
\hline Perspectivas em Ciência da Informação & Brasil & 1 \\
\hline Publications & Suíça & 1 \\
\hline Transinformação & Brasil & 1 \\
\hline
\end{tabular}

Fonte: dados da pesquisa, 2021.

E reunimos os dados dos países mais produtivos, na busca realizada.

Quadro 8 - Países mais produtivos

\begin{tabular}{|c|c|}
\hline País & Quantitativo \\
\hline Espanha & 5 \\
\hline Brasil & 4 \\
\hline Dinamarca & 1 \\
\hline Alemanha & 1 \\
\hline Itália & 1 \\
\hline EUA & 1 \\
\hline
\end{tabular}

Fonte: dados da pesquisa, 2021. 
Mais uma vez, utilizamos a estratégia de busca na base de dados WoS. Mantendo o mesmo recorte temporal e a mesma categoria, porém, dessa vez utilizando o argumento de busca Periódico Científico "Scientific Journals", recuperamos 469 resultados, dentre os quais, ao utilizarmos o termo "Social Media" para filtrar, encontramos 17 documentos. Em relação aos termos que mais aparecem nos descritores dos documentos recuperados, identificamos "altmetrics" e variados, "visibility", "open access" e "algorithm". Nesses resultados, Espanha e Brasil mais uma vez se destacam, com cinco e quatro resultados, respectivamente, seguidos pela Finlândia, com três resultados, e pela Venezuela, com dois.

\section{Quadro 9 - Países mais produtivos}

\begin{tabular}{|c|c|}
\hline País & Quantitativo \\
\hline Espanha & 5 \\
\hline Brasil & 4 \\
\hline Finlândia & 3 \\
\hline Venezuela & 2 \\
\hline Áustria & 1 \\
\hline Canadá & 1 \\
\hline Dinamarca & 1 \\
\hline Hungria & 1 \\
\hline México & 1 \\
\hline Portugal & 1 \\
\hline Coréia do Sul & 1 \\
\hline
\end{tabular}

Fonte: Dados da pesquisa, 2021.

Em relação aos autores, encontramos aqui outro caso em que se encontram em destaque dois autores que colaboraram e apresentaram dois resultados, cada: Katvala, M. e Kortelainen, T., ambos filiados à University of Oulu, da Finlândia. Os trabalhos foram publicados nos periódicos Journal of Informetrics, dos Países Baixos e Information Research - An International Electronic Journal, da Inglaterra. Entre os jornais, destacam-se o Profesional de 
La Información, da Espanha, com quatro resultados e Online Information Review, da Inglaterra, e Scientometrics, dos Países Baixos, com 2 resultados cada.

Quadro 10 - Revistas mais produtivas

\begin{tabular}{|l|c|c|}
\hline \multicolumn{1}{|c|}{ Título da publicação } & País & Quantitativo \\
\hline Profesional de La Información & Espanha & 4 \\
\hline Online Information Review & Inglaterra & 2 \\
\hline Scientometrics & Países Baixos & 2 \\
\hline E Ciencias De La Información & Venezuela & 1 \\
\hline Education For Information & Dinamarca & 1 \\
\hline Em Questão & Brasil & 1 \\
\hline $\begin{array}{l}\text { Encontros Bibli - Ver. Eletr. de Biblio. e } \\
\text { Ci. da Inf. }\end{array}$ & Brasil & 1 \\
\hline Informacios Tarsadalom & Hungria & 1 \\
\hline $\begin{array}{l}\text { Information Research An International } \\
\text { Electronic Journal }\end{array}$ & Inglaterra & 1 \\
\hline Investigacion Bibliotecologica & México & 1 \\
\hline Journal of Informetrics & Países Baixos & 1 \\
\hline Transinformação & Brasil & 1 \\
\hline
\end{tabular}

Fonte: Dados da pesquisa, 2021.

Este último quadro sintetiza a distribuição da produção por periódicos e respectivos países, com destaque para Profesional de La Información, publicado na Espanha. Para visualização dos resultados a partir dos descritores, apresentamos uma nuvem de palavras como resultado da extração das palavras-chave de todos os artigos recuperados na pesquisa. 


\section{Figura 4 - Nuvem de termos representativos dos artigos recuperados}

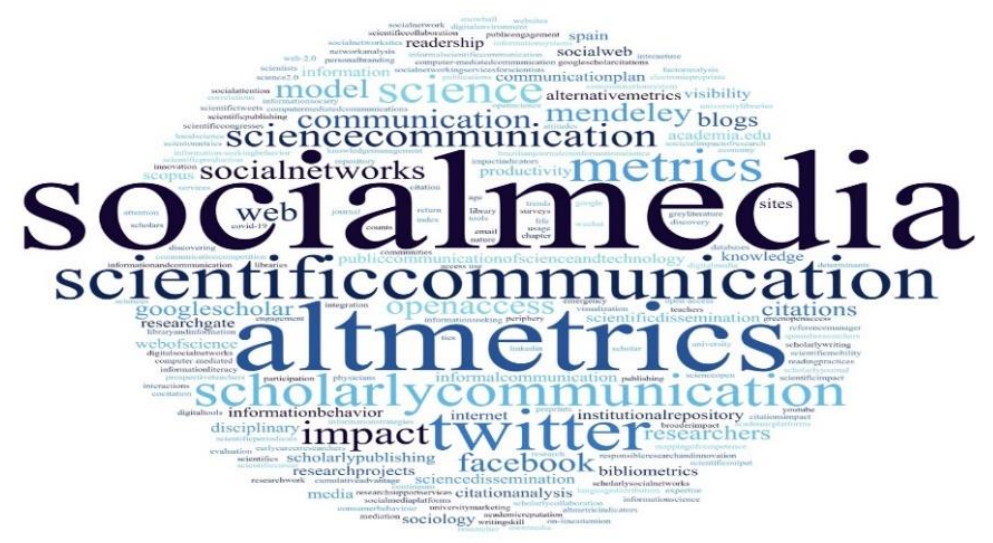

Fonte: Dados da pesquisa, 2021.

Essa nuvem possibilita observar os conceitos presentes nos artigos recuperados na pesquisa, suas relações temáticas e nível de relevância. É importante para compreender os termos que apresentam relações de proximidade temática e epistemológica. Destarte, apresenta um caminho para pesquisas que tenham como interesse a comunicação científica e os conceitos que estão relacionados.

\section{CONSIDERAÇÕES FINAIS}

O sistema de comunicação científica é historicamente consolidado, mas está sendo modificado pelas tecnologías digitais de informação e comunicação, que apresentam novas formas para produção, organização e, principalmente, comunicação de informação científica. Estamos caminhando, embora ainda no início, no sentido de incorporar as redes sociais digitais de pesquisa aos sistemas clássicos de comunicação científica. Trata-se da evolução natural de um sistema pensado e construido ao longo de 300 anos.

Essa convergência de canais de comunicação (blog, Facebook, Twitter, Instagram), forma uma rede de canais de comunicação cujo impacto ainda não conseguimos mensurar totalmente, mas conseguimos imaginar que levarão a comunicação científica a patamares bem diferentes do que conhecemos hoje. $O$ 
impacto de um artigo científico não deverá ser observado apenas pela contribuição à área em que é produzido, mas também por seu alcance em toda a sociedade. E, se vivemos na sociedade em rede, onde a conexão é a regra, devemos também observar o alcance das pesquisas publicizadas através dos artigos compartilhados nas rede sociais.

Nesse sentido, as redes sociais digitais de periódicos científicos podem ser entendidas como estratégias para facilitar o compartilhamento de informações de forma mais direta e próxima, não só para os pares, mas para toda a sociedade.

O processo de comunicação científica continua em evolução, incorporando inovações que vão muito além dos aspectos tecnológicos. As inovações observadas neste processo têm um elevado nível de penetrabilidade e perpassam todo o regime de informação da comunicação científica, especialmente com relação aos atores sociais e aos artefatos sintáticos e semânticos de informação. Trata-se de cenário desafiador, especialmente para aqueles que atuam nas atividades relacionadas à gestão e comunicação da informação.

Nesse cenário, pesquisas sobre comunicação científica estão sendo desenvolvidas em todo o mundo, buscando apresentar soluções e compreender esse processo de mudança. A Ciência da Informação e seus pesquisadores têm muito a contribuir, colaborando na construção de uma ampla rede de pesquisas relacionadas com a temática. Afinal o protagonismo das atividades científicas na sociedade contemporânea, em que o regime de informação é hegemônico, é um novo paradigma, e a comunicação científica é consequência desse protagonismo e da própria atividade científica. Certamente os pesquisadores da área de Ciência da Informação terão um relevante papel a desempenhar, nesse processo.

\section{REFERÊNCIAS}

BRAMAN, S. The emergent global information policy regime, In: BRAMAN, S. (ed.) The emergent global information policy regime. Hampshire, Palgrave, 2004. 
CAREGNATO, S.E. SOUZA, S.C de. Blogs científicos.br?: um estudo exploratório. Informação \& Informação, Londrina, v.15, n. esp., p. 56-75, 2010. DOI: 10.5433/1981-8920.2010v15nesp.p56.

CASTELLS, M. O poder da comunicação. 1. ed. Rio de Janeiro: Paz e Terra, 2015.

CLARIVATE ANALYTICS. Web of Science. [S.I.]: Clarivate Analytics, c2021. Disponível em: https://www.webofscience.com. Acesso em: 11 set. 2021.

FREIRE, G. H. de A.; FREIRE, I. M. A literatura cinzenta se ilumina na web. Informação \& Sociedade: Estudos, v. 25, n. 2, p. 5-6, maio/ago. 2015. Disponível em:

https://periodicos.ufpb.br/ojs2/index.php/ies/article/view/5/13740. Acesso em: 11 set. 2021.

FROHMANN, B. Taking policy beyond Information Science: applying the actor network theory for connectedness: information, systems, people, organizations. In: ANNUAL CONFERENCE CANADIAN ASSOCIATION FOR INFORMATION SCIENCE, 23., 1995. Proceedings [...]. Edmond, Alberta, 1995.

GONZÁLEZ DE GÓMEZ, M. N. O caráter seletivo das ações de informação. INFORMARE - Cadernos do Programa de Pós-Graduação em Ciência da Informação, Rio de Janeiro, v. 5, n. 2, p. 7-30, jul./dez, 1999.

LARIVIÈRE, V., HAUSTEIN, S., MONGEON, P. The Oligopoly of Academic Publishers in the Digital Era. PloS ONE [online], v. 10, n. 6, jun. 2015. DOI: 10.1371/journal.pone.0127502. Disponível em: http://journals.plos.org/plosone/article?id=10.1371/journal.pone.0127502. Acesso em: 4 jul. 2018.

MARTELETO, R. M. Redes Sociais, Mediação e Apropriação de Informações: situando campos, objetos e conceitos na pesquisa em Ciência da Informação. Tendências da Pesquisa Brasileira em Ciência da Informação, v. 3, n. 1, p. 27-46, jan./dez. 2010. Disponível em:

https://www.arca.fiocruz.br/handle/icict/2247. Acesso em: 11 set. 2021.

MEADOWS, A. J. A comunicação científica. Trad. Antonio Agenor Briquet de Lemos. Brasília: Briquet de Lemos, 1999.

OLIVEIRA, T. Midiatização da Ciência: Reconfiguração do paradigma da comunicação científica na era digital. In: ENCONTRO ANUAL DA COMPÓS, 27., 2018, Belo Horizonte. Anais [...]. Compós: Belo Horizonte, 2018. Disponível em: http://www.compos.org.br/data/arquivos_2018/trabalhos_arquivo_E2914S5R8A UHF69PEX0R_27_6978_27_02_2018_09_23_29.pdf. Acesso em: 4 jul. 2018.

SANTILLANA, G. di. O historiador e a teoria da informação. In: 0 conceito de informação na ciência contemporânea. Rio de Janeiro: Paz e Terra, 1970. 
SANTOS, M. Metamorfoses do espaço habitado. São Paulo: Hucitec, 1997.

SENA, A.; PIRES, E. A. de N. Qualidade da informação: uma breve abordagem sobre a contribuição do periódico científico para ciência. Múltiplos olhares em Ciência da Informação, v. 2, n. 1, mar. 2012. Disponível em: http://hdl.handle.net/20.500.11959/brapci/61867. Acesso em: 6 maio 2021.

STUMPF, I. R. C. Passado e futuro das revistas científicas. Ciência da Informação, v. 25, n. 3, 1996. Disponível em: http://revista.ibict.br/ciinf/article/view/637. Acesso em: 6 maio 2021.

WERSIG, G. Information Science: The study of postmodern knowledge usage. Information Processing and Management, v. 29, n. 2, p. 229-239, 1993. Disponível em: https://www.sciencedirect.com/science/article/abs/pii/030645739390006Y. Acesso em: 6 maio 2021.

WERSIG, G., NEVELING, U. The phenomena of interest to information science. The Information Scientist, v. 9, n. 4, 1975.

\title{
THE SCIENTIFIC COMMUNICATION INFORMATION REGIME: AN APPROACH
}

\begin{abstract}
:
Objectives: Its general objective is to identify the literature that addresses information actions developed in cyberspace through social networks of periodicals for communication and dissemination of scientific knowledge to society. The specific objectives are: to map scientific journals in the Information Science area that have social networks; describe the information regime of scientific journals and their respective social networks; identify information policies aimed at the use of social networks as a marketing tool for periodicals. Methodology: The research is exploratory, descriptive, and qualitative in nature, using documentary research techniques, organizing the results from categories to achieve the outlined objectives. Expected results: It is expected to provide a contribution to the enrichment of thematic studies in Information Science, as well as the understanding of how the process of communication and scientific dissemination is using this new communication channel, in the technical, social and economic dimensions. This process may reveal possibilities, limitations and future applications of digital social networks for scientific journals, not only in the context of Information Science but also in other areas of scientific knowledge.
\end{abstract}

Descriptors: Scientific communication. Scientific divulgation. Digital social networks. Literature review.

\section{EL RÉGIME DE INFORMACIÓN DE COMUNICACIÓN CIENTÍFICA: UN ENFOQUE}




\section{RESUMEN}

Objetivos: Su objetivo general es identificar la literatura que aborde las acciones de información desarrolladas en el ciberespacio a través de las redes sociales de revistas para la comunicación y difusión del conocimiento científico a la sociedad. Los objetivos específicos son: mapear revistas científicas del área de Ciencias de la Información que cuenten con redes sociales; describir el régimen de información de las revistas científicas y sus respectivas redes sociales; Identificar políticas de información orientadas al uso de las redes sociales como herramienta de marketing de publicaciones periódicas. Metodología: La investigación es de naturaleza exploratoria, descriptiva y cualitativa, utilizando técnicas de investigación documental, organizando los resultados por categorías para lograr los objetivos planteados.

Resultados esperados: Se espera que aporte un aporte al enriquecimiento de los estudios temáticos en Ciencias de la Información, así como a la comprensión de cómo el proceso de comunicación y divulgación científica está utilizando este nuevo canal de comunicación, en las dimensiones técnica, social y económica. Este proceso puede revelar posibilidades, limitaciones y futuras aplicaciones de las redes sociales digitales para revistas científicas, no solo en el contexto de las Ciencias de la Información sino también en otras áreas del conocimiento científico.

Descriptores: Comunicación científica. Divulgación científica. Redes sociales digitales. Revisión de literatura.

Recebido em: 15.10 .2021

Aceito em: 21.10.2021 